\title{
The role of hyperfine mixing in $b \rightarrow c$ semileptonic decays of doubly-heavy baryons
}

\author{
$\begin{array}{lll}\text { C. Albertus } & 1,2 ; 1) & \text { E. Hernández } \\ & \text { Ji2) } & \text { Jieves } \\ & 2 ; 3)\end{array}$ \\ 1 (Departamento de Física Fundamental e IUFFyM, Universidad de Salamanca, E-37008 Salamanca, Spain) \\ 2 (Instituto de Física Corpuscular (IFIC), Centro Mixto CSIC-Universidad de Valencia, Institutos de Investigación de Paterna, \\ Aptd. 22085, E-46071 Valencia, Spain)
}

\begin{abstract}
We analyze the effects of hyperfine mixing in $b \rightarrow c$ semileptonic decays of doubly heavy baryons. We qualitatively confirm the results by W. Roberts and M. Pervin in Int. J. Mod. Phys. A, 2009, 24: 24012413 , finding that mixing has a great impact on those transitions. However, predictions without mixing differ by a factor of 2 and this discrepancy translates to the mixed case where large differences in decay widths are observed between the two calculations.
\end{abstract}

Key words doubly-heavy baryons, semileptonic decay, hyperfine mixing

PACS 12.39.Jh, 13.30.Ce

\section{Introduction}

According to heavy quark spin symmetry, in the infinite heavy quark mass limit, one can select the total spin $\left(S_{h}\right)$ of the heavy quark subsystem of a dou- bly heavy baryon to be well defined ${ }^{[1]}$. This result has been used as a basis for the usual classification scheme of doubly heavy baryons. In Table 1 we list the ground state doubly heavy baryons with quantum numbers $J^{\pi}=\frac{1}{2}^{+}, \frac{3}{2}^{+}$that are included in this study.

Table 1. Quantum numbers and quark content of doubly heavy baryons

\begin{tabular}{|c|c|c|c|c|c|c|c|}
\hline Baryon & $\begin{array}{c}\text { Quark content } \\
(\mathrm{l}=\mathrm{u}, \mathrm{d})\end{array}$ & $S_{h}$ & $J^{\pi}$ & Baryon & Quark content & $S_{h}$ & $J^{\pi}$ \\
\hline$\Xi_{c c}$ & $\{\mathrm{c} c \mathrm{c}\} \mathrm{l}$ & 1 & $1 / 2^{+}$ & $\bar{\Omega} \Omega_{c c}$ & $\{c c\} s$ & 1 & $\frac{1 / 2^{+}}{}$ \\
\hline$\Xi_{c c}^{*}$ & $\left\{\begin{array}{cc}c & c\end{array}\right]$ & 1 & $3 / 2^{+}$ & $\Omega_{c c}^{*}$ & $\{c c\} s$ & 1 & $3 / 2^{+}$ \\
\hline$\Xi_{b b}$ & $\{b \quad b\} l$ & 1 & $1 / 2^{+}$ & $\Omega_{b b}$ & $\{b$ b $\} s$ & 1 & $1 / 2^{+}$ \\
\hline$\Xi_{b b}^{*}$ & $\{b \quad b\} l$ & 1 & $3 / 2^{+}$ & $\Omega_{b b}^{*}$ & $\{b \mathrm{~b}\} \mathrm{s}$ & 1 & $3 / 2^{+}$ \\
\hline$\Xi_{b c}$ & $\{b$ c $\}$ l & 1 & $1 / 2^{+}$ & $\Omega_{b c}$ & $\{b \mathrm{~b}\} \mathrm{s}$ & 1 & $1 / 2^{+}$ \\
\hline$\Xi_{b c}^{*}$ & $\{b \mathrm{c}\} \mathrm{l}$ & 1 & $3 / 2^{+}$ & $\Omega_{b c}^{*}$ & $\{b \mathrm{c}\} \mathrm{s}$ & 1 & $3 / 2^{+}$ \\
\hline$\Xi_{b c}^{\prime}$ & {$[\mathrm{b} c \mathrm{c}] \mathrm{l}$} & 0 & $1 / 2^{+}$ & $\Omega_{b c}^{\prime}$ & {$\left[\begin{array}{ll}b & c\end{array}\right] \mathrm{s}$} & 0 & $1 / 2^{+}$ \\
\hline
\end{tabular}

Hyperfine interaction between the light and any of the heavy quarks can admix components with both $S_{h}=0$ and $S_{h}=1$ in the wave function. The mixing should be very small in the $b b$ and $c c$ sectors as it will imply higher radial excitations or larger angular momentum. However, mixing could be particularly important for baryons with $b c$ heavy quark content where one expects the actual physical $\Xi(\Omega)$ states to be admixtures of the $\Xi_{b c}, \Xi_{b c}^{\prime}\left(\Omega_{b c}, \Omega_{b c}^{\prime}\right)$ ones given in
Table 1 This mixing gives rise to small changes in the masses but, as suggested in Ref. ${ }^{[2]}$, it could have a great impact on the widths of decays involving those states. This has been investigated in Ref. $\frac{[3]}{3}$ using harmonic oscillator wave functions as an expanding basis. Here we shall try to confirm their findings using our variational wave functions described in Ref. ${ }^{[4]}$ and obtained with the use of the AL1 potential of Ref. ${ }^{[\underline{5}}$. All the details on the calculations can be found in

* Supported by DGI and FEDER funds, under contracts FIS2008-01143/FIS, FIS2006-03438, FPA2007-65748, CSD2007-00042, by Junta de Castilla y León under contracts SA016A07 and GR12, and by the EU HadronPhysics2 project

1) E-mail: albertus@usal.es

2) E-mail: gajatee@usal.es

3) E-mail:jmnieves@ific.uv.es

(C) 2009 Chinese Physical Society and the Institute of High Energy Physics of the Chinese Academy of Sciences and the Institute of Modern Physics of the Chinese Academy of Sciences and IOP Publishing Ltd 
Ref. ${ }^{[6]}$ and references therein.

\section{Results and discussion}

\subsection{Masses for unmixed states}

Our results for the masses are given in Table 2. We compare them with the results obtained in Ref. ${ }^{[7]}$ using a relativistic quark model that assumes a light quark-heavy diquark structure, and in the above mentioned Ref. ${ }^{[2]}$ where they use a nonrelativistic approach with harmonic oscillator wave func- tions. The agreement with the calculation in Ref. ${ }^{[7]}$ is very good for $\Xi$ baryons whereas for $\Omega$ baryons their masses are some $50 \sim 90 \mathrm{MeV}$ larger. The masses obtained in Ref. ${ }^{[2]}$ are always larger than ours by $50 \sim 180 \mathrm{MeV}$. On the experimental side the SELEX Collaboration claimed evidence for the $\Xi_{c c}^{+}$baryon, in the $\Lambda_{c}^{+} K^{-} \pi^{+}$and $p D^{+} K^{-}$decay modes, with a mass of $M_{\Xi_{c c}^{+}}=3519 \pm 1 \mathrm{MeV} / \mathrm{c}^{2}{ }^{[8]}$, a $100 \mathrm{MeV}$ smaller than most theoretical predictions. No other experimental collaboration has found evidence for doubly charmed baryons so far and, at present, the $\Xi_{c c}^{+}$has only a one star status.

Table 2. Masses (in MeV) for unmixed states

\begin{tabular}{lccc||cccc}
\hline & This work & {$[7]$} & {$[2]$} & & This work & {$[7]$} & {$[2]$} \\
\hline$M_{\Xi_{c c}}$ & 3613 & 3620 & 3676 & $M_{\Omega_{c c}}$ & 3712 & 3778 & 3815 \\
$M_{\Xi_{c c}^{*}}$ & 3707 & 3727 & 3753 & $M_{\Omega_{c c}^{*}}$ & 3795 & 3872 & 3876 \\
$M_{\Xi_{b b}}$ & 10198 & 10202 & 10340 & $M_{\Omega_{b b}}$ & 10269 & 10359 & 10454 \\
$M_{\Xi_{b b}^{*}}$ & 10237 & 10237 & 10367 & $M_{\Omega_{b b}^{*}}$ & 10307 & 70389 & 10486 \\
$M_{\Xi_{b c}}$ & 6928 & 6933 & 7020 & $M_{\Omega_{b c}}$ & 7013 & 7088 & 7147 \\
$M_{\Xi_{b c}^{\prime}}$ & 6958 & 6963 & 7044 & $M_{\Omega_{b c}^{\prime}}$ & 7038 & 7116 & 7166 \\
$M_{\Xi_{b c}^{*}}$ & 6996 & 6980 & 7078 & $M_{\Omega_{b c}^{*}}$ & 7075 & 7130 & 7191 \\
\hline
\end{tabular}

\subsection{Decay widths for unmixed states}

Our model to evaluate $b \rightarrow c$ semileptonic decays of doubly heavy baryons is described in Ref. ${ }^{[4]}$. We use a spectator approximation in which any of the $b$ quarks in the initial state can decay into any of the $c$ quarks in the final state. This, together with the right normalization for baryon states containing two equal heavy quarks, gives an extra factor $\sqrt{2}$ in the transition amplitude when compared to the similar $b \rightarrow c$ decay in baryons with just one heavy quark.

The results that we obtain are shown in Table 3 where for comparison we also show the results in Refs. $\left.{ }^{[9}, 10\right]$, obtained within different relativistic approaches, and in the nonrelativistic calculation of
Ref. ${ }^{[3]}$. Our results are in a global fair agreement with the ones in Ref. ${ }^{[9]}$. As for the other relativistic calculation in Ref. ${ }^{[10]}$, the agreement is fair for transitions with a $b c$ baryon in the initial state but there is an approximate factor of 2 discrepancy for transitions with a $b c$ baryon in the final state. The nonrelativistic calculation in Ref. ${ }^{[3]}$ also gives results that are roughly a factor of 2 smaller than ours for all decays. A very interesting feature of the decay widths shown in Table 3 is that they are very different for transitions involving $\Xi_{b c}$ or $\Xi_{b c}^{\prime}\left(\Omega_{b c}\right.$ or $\left.\Omega_{b c}^{\prime}\right)$. This means, as suggested in Ref. ${ }^{[2]}$, that mixing in those states, provided the admixture coefficients are large, can have a great impact on the decay widths.

Table 3. Semileptonic decay widths (in $10^{-14} \mathrm{GeV}$ ) for unmixed states. We use $\left|V_{c b}\right|=0.0413 . l=e, \mu$

\begin{tabular}{lcccc||ccccc}
\hline & This work & $\underline{\underline{9}]}$ & $\underline{10}]$ & $\underline{\underline{3}]}$ & & This work & $\underline{[9]}$ & {$[10]$} \\
\hline$\Gamma\left(\Xi_{b b}^{*} \rightarrow \Xi_{b c}^{\prime} l \bar{\nu}_{l}\right)$ & 1.08 & 0.82 & $0.36 \pm 0.10$ & - & $\Gamma\left(\Omega_{b b}^{*} \rightarrow \Omega_{b c}^{\prime} l \bar{\nu}_{l}\right)$ & 1.14 & 0.85 & $0.42 \pm 0.14$ & - \\
$\Gamma\left(\Xi_{b b}^{*} \rightarrow \Xi_{b c} l \bar{\nu}_{l}\right)$ & 0.36 & 0.28 & $0.14 \pm 0.04$ & - & $\Gamma\left(\Omega_{b b}^{*} \rightarrow \Omega_{b c} l \bar{\nu}_{l}\right)$ & 0.38 & 0.29 & $0.15 \pm 0.05$ & - \\
$\Gamma\left(\Xi_{b b} \rightarrow \Xi_{b c}^{\prime} l \bar{\nu}_{l}\right)$ & 1.09 & 0.82 & $0.43 \pm 0.12$ & 0.41 & $\Gamma\left(\Omega_{b b} \rightarrow \Omega_{b c}^{\prime} l \bar{\nu}_{l}\right)$ & 1.16 & 0.83 & $0.48 \pm 0.12$ & 0.51 \\
$\Gamma\left(\Xi_{b b} \rightarrow \Xi_{b c} l \bar{\nu}_{l}\right)$ & 2.00 & 1.63 & $0.80 \pm 0.30$ & 0.69 & $\Gamma\left(\Omega_{b b} \rightarrow \Omega_{b c} l \bar{\nu}_{l}\right)$ & 2.15 & 1.70 & $0.86 \pm 0.32$ & 0.92 \\
$\Gamma\left(\Xi_{b c}^{\prime} \rightarrow \Xi_{c c} l \bar{\nu}_{l}\right)$ & 1.36 & 0.88 & $1.10 \pm 0.32$ & - & $\Gamma\left(\Omega_{b c}^{\prime} \rightarrow \Omega_{c c} l \bar{\nu}_{l}\right)$ & 1.36 & 0.95 & $0.98 \pm 0.28$ & - \\
$\Gamma\left(\Xi_{b c} \rightarrow \Xi_{c c} l \bar{\nu}_{l}\right)$ & 2.57 & 2.30 & $2.10 \pm 0.70$ & 1.38 & $\Gamma\left(\Omega_{b c} \rightarrow \Omega_{c c} l \bar{\nu}_{l}\right)$ & 2.58 & 2.48 & $1.88 \pm 0.62$ & 1.54 \\
$\Gamma\left(\Xi_{b c}^{\prime} \rightarrow \Xi_{c c}^{*} l \bar{\nu}_{l}\right)$ & 2.35 & 1.70 & $2.01 \pm 0.62$ & - & $\Gamma\left(\Omega_{b c}^{\prime} \rightarrow \Omega_{c c}^{*} l \bar{\nu}_{l}\right)$ & 2.35 & 1.83 & $1.93 \pm 0.60$ & - \\
$\Gamma\left(\Xi_{b c} \rightarrow \Xi_{c c}^{*} l \bar{\nu}_{l}\right)$ & 0.75 & 0.72 & $0.64 \pm 0.19$ & 0.52 & $\Gamma\left(\Omega_{b c} \rightarrow \Omega_{c c}^{*} l \bar{\nu}_{l}\right)$ & 0.76 & 0.74 & $0.62 \pm 0.19$ & 0.56 \\
\hline
\end{tabular}




\subsection{Results with mixing}

We obtain the mixed $b c$ states by diagonalization of the corresponding mass matrices. In our calculation the mixed states and masses are given by

$$
\begin{aligned}
& \Xi_{b c}^{(1)}=0.902 \Xi_{b c}^{\prime}+0.431 \Xi_{b c}, M_{\Xi_{b c}^{(1)}}=6967 \mathrm{MeV}, \\
& \Xi_{b c}^{(2)}=-0.431 \Xi_{b c}^{\prime}+0.902 \Xi_{b c}, M_{\Xi_{b c}^{(2)}}=6919 \mathrm{MeV}, \\
& \Omega_{b c}^{(1)}=0.899 \Omega_{b c}^{\prime}+0.437 \Omega_{b c}, M_{\Omega_{b c}^{(1)}}=7046 \mathrm{MeV}, \\
& \Omega_{b c}^{(2)}=-0.437 \Omega_{b c}^{\prime}+0.899 \Omega_{b c}, M_{\Omega_{b c}^{(2)}}=7005 \mathrm{MeV} .
\end{aligned}
$$

By comparison to the unmixed results shown in Table 2, we see the masses change but very little when mixing is taken in to account. However, as shown in Eq.(1), the admixture is important and it can affect the decay widths.

Note that these mixed states are close to the states (in the what follows $B \equiv \Xi, \Omega$ )

$$
\begin{aligned}
& B_{b c}^{(1)} \approx\left(|q c ; 1\rangle \otimes\left|b ; \frac{1}{2}\right\rangle\right)^{J=1 / 2} \equiv \frac{\sqrt{3}}{2} B_{b c}^{\prime}+\frac{1}{2} B_{b c} \\
& B_{b c}^{(2)} \approx\left(|q c ; 0\rangle \otimes\left|b ; \frac{1}{2}\right\rangle\right)^{J=1 / 2} \equiv-\frac{1}{2} B_{b c}^{\prime}+\frac{\sqrt{3}}{2} B_{b c}
\end{aligned}
$$

in which the light and the $c$ quark couple to well defined spin 1 or 0 .

Table 4. Semileptonic decay widths (in $10^{-14} \mathrm{GeV}$ ) for mixed states. We use $\left|V_{c b}\right|=0.0413 . l=e, \mu$

\begin{tabular}{lcc||ccc}
\hline & This work & $\underline{\underline{3}]}$ & & This work \\
\hline$\Gamma\left(\Xi_{b b}^{*} \rightarrow \Xi_{b c}^{(1)} l \bar{\nu}_{l}\right)$ & 0.47 & - & $\Gamma\left(\Omega_{b b}^{*} \rightarrow \Omega_{b c}^{(1)} l \bar{\nu}_{l}\right)$ & 0.48 \\
$\Gamma\left(\Xi_{b b}^{*} \rightarrow \Xi_{b c}^{(2)} l \bar{\nu}_{l}\right)$ & 0.99 & - & $\Gamma\left(\Omega_{b b}^{*} \rightarrow \Omega_{b c}^{(2)} l \bar{\nu}_{l}\right)$ & 1.06 & - \\
$\Gamma\left(\Xi_{b b} \rightarrow \Xi_{b c}^{(1)} l \bar{\nu}_{l}\right)$ & 2.21 & 0.95 & $\Gamma\left(\Omega_{b b} \rightarrow \Omega_{b c}^{(1)} l \bar{\nu}_{l}\right)$ & 2.36 & 0.99 \\
$\Gamma\left(\Xi_{b b} \rightarrow \Xi_{b c}^{(2)} l \bar{\nu}_{l}\right)$ & 0.85 & 0.33 & $\Gamma\left(\Omega_{b b} \rightarrow \Omega_{b c}^{(2)} l \bar{\nu}_{l}\right)$ & 0.91 & 0.30 \\
$\Gamma\left(\Xi_{b c}^{(1)} \rightarrow \Xi_{c c} l \bar{\nu}_{l}\right)$ & 0.38 & - & $\Gamma\left(\Omega_{b c}^{(1)} \rightarrow \Omega_{c c} l \bar{\nu}_{l}\right)$ & 0.37 & - \\
$\Gamma\left(\Xi_{b c}^{(2)} \rightarrow \Xi_{c c} l \bar{\nu}_{l}\right)$ & 3.51 & 1.92 & $\Gamma\left(\Omega_{b c}^{(2)} \rightarrow \Omega_{c c} l \bar{\nu}_{l}\right)$ & 3.52 & - \\
$\Gamma\left(\Xi_{b c}^{(1)} \rightarrow \Xi_{c c}^{*} l \bar{\nu}_{l}\right)$ & 3.14 & - & $\Gamma\left(\Omega_{b c}^{(1)} \rightarrow \Omega_{c c}^{*} l \bar{\nu}_{l}\right)$ & 3.14 & 0.013 \\
$\Gamma\left(\Xi_{b c}^{(2)} \rightarrow \Xi_{c c}^{*} l \bar{\nu}_{l}\right)$ & 0.017 & 0.026 & $\Gamma\left(\Omega_{b c}^{(2)} \rightarrow \Omega_{c c}^{*} l \bar{\nu}_{l}\right)$ & 0.014 & \\
\hline
\end{tabular}

The new decay widths involving the mixed states $\Xi_{b c}^{(1)}, \Xi_{b c}^{(2)}$ and $\Omega_{b c}^{(1)}, \Omega_{b c}^{(2)}$ are now given in Table 4 We see rather big changes from the values in Table 3 where unmixed states were used. Special attention deserves the $B_{b c}^{(2)} \rightarrow B_{c c}^{*}$ transitions where the width reduces by a large factor of 44 (54) for the $\Xi_{b c}^{(2)} \rightarrow \Xi_{c c}^{*}\left(\Omega_{b c}^{(2)} \rightarrow \Omega_{c c}^{*}\right)$ decay compared to the unmixed case. This can be easily understood by taking into account that $B_{b c}^{(2)} \approx\left(|q c ; 0\rangle \otimes\left|b ; \frac{1}{2}\right\rangle\right)^{J=1 / 2}$. In the latter state the light and $c$ quarks are coupled to spin 0 , whereas in the $B_{c c}^{*}$ the light and any of the $c$ quarks are in a relative spin 1 state. In any spectator calculation, as the ones here and in Ref. ${ }^{[3]}$, the amplitude for the $\left(|q c ; 0\rangle \otimes\left|b ; \frac{1}{2}\right\rangle\right)^{J=1 / 2} \rightarrow B_{c c}^{*}$ transition cancels due to the orthogonality of the different spin states of the spectator quarks in the initial and final baryons. The fact that $B_{b c}^{(2)}$ slightly deviates from $\left(|q c ; 0\rangle \otimes\left|b ; \frac{1}{2}\right\rangle\right)^{J=1 / 2}$ produces a non zero, but small, decay width.

\section{Conclusions}

We qualitatively confirm the findings in Ref. ${ }^{[3]}$ as to the relevance of hyperfine mixing in $b \rightarrow c$ semileptonic decays of doubly heavy baryons. On the other hand the absolute predictions are quite differ- ent. This is a reflection of the approximate factor of 2 difference we already found in Table 3 for unmixed states.

C. A. acknowledges a contract supported by PIECSIC $200850 I 238$ during his stay at IFIC.

\section{References}

1 JENKINS E, LUKE M, MANOHAR A V et al., Nuc. Phys. B, 1993, 390: 463-473

2 ROBERTS W, PERVIN M, Int. J. Mod. Phys. A, 2008, 23: $2817-2860$

3 ROBERTS W, PERVIN M, Int. J. Mod. Phys. A, 2009, 24: $2401-2413$

4 ALBERTUS C, HERNÁNDEZ E, NIEVES J et al., Eur. Phys. J. A., 2007, 32: 183-199; erratum ibid Eur. Phys. J. A, 2008, 36: 119

5 SEMAY C, SILVESTRE-BRAC B, Z. Phys. C, 1994, 61: $271-275$

6 ALBERTUS C,HERNÁNDEZ E, NIEVES J arXiv: 0911.0889

7 EBERT D, FAUSTOV R N, GALKIN V O et al. Phy. Rev D, 2002, 66: 014008

8 MATTSON M, ALKHAZOV G, ATAMANTCHOUK A G et al. (SELEX Collaboration), Phys. Rev. Lett., 2002, 89: 112001

9 EBERT D, FAUSTOV R N, GALKIN V O et al., Phy. Rev D, 2004, 70: 014018; erratum ibid Phys. Rev. D, 2008, 77: 079903

10 FAESSLER A, GUTSCHE TH, IVANOV M A et al., Phys. Rev. D, 2009, 80: 034025 